\title{
Coordination in Catalan Sign Language: a syntactic account for conjunction
}

\author{
Giorgia Zorzi (UPF, Universitat Pompeu Fabra) \\ giorgia.zorzi@upf.edu
}

\begin{abstract}
Coordination is a syntactic structure that is attested and productive in both spoken and signed languages. In sign languages, this structure is primarily expressed asyndetically, that is not using manual markers, but rather non-manual ones (NMMs), such as body and head turns, to mark the conjuncts. This paper aims to describe the expression of coordination in Catalan Sign Language (LSC), focusing on TP conjunction, and present the main tests used to identify this type of structure. Building on the analyses proposed until now for spoken languages, the objective is to provide a syntactic analysis for conjunction in LSC. Considerations on the directionality of the structure will contribute to explaining some of the properties of coordination in LSC such as the ungrammaticality of both Across-the-Board (ATB) extraction and wide scope of negation. Moreover, the adoption of Coordination Phrase (CoP) as the syntactic category for coordination provides a structure that can also be extended to other types of coordination, such as disjunctive and adversative.
\end{abstract}

Keywords: coordination, TP conjunction, syntax, Catalan Sign Language (LSC), CoP

\section{Introduction}

Coordination is defined by Haspelmath (2007) as a syntactic construction in which two or more units of the same type are combined into a larger unit and still have the same semantic relations with other surrounding elements. Several authors, though, among which Sag et al. (1985) and Munn (1993), point out that it is possible to combine different syntactic categories. We can see a few examples in (1). In (1a) two nominal phrases (NPs) are coordinated while in (1b, c) the coordinated constituents are, respectively, an adjectival phrase (AP) and a NP, and a prepositional phrase (PP) and a complementizer phrase (CP). (1b) and (1c) are from Sag et al. (1985, 143) and Munn $(1993,80)$, respectively.

(1) a. [NP Mary] and [NP John] won the game.

b. Jermaine is [AP boring] and [NP a fool].

c. You can depend [PP on my assistant] and [CP that he will be on time].

There are three main types of coordination depending on the coordinator that is used: conjunctive, in which the coordinator is "and", like in (2a), disjunctive, in which "or" is the one used, like in (2b), and adversative, in which we find the coordinator "but", like in (2c).

(2) a. Snowwhite ate and drank.

b. She was a countess or a princess.

c. The dwarfs were ugly but kind. (Haspelmath 2007, 2) 
This paper, though, will only focus on sentential conjunction of tense phrases (TPs), ${ }^{1}$ like in example (3).

(3) [TP Mary went to school] and [TP John had lunch].

Among the types of coordination, it is also possible to distinguish between syndetic and asyndetic coordination, depending on the coordinator being expressed or not. In syndetic coordination, the coordinator is overt while in the asyndetic one, also called juxtaposition, the intonation is the only way to signal the presence of coordination, as we can see in (4).

(4) I came, I saw, I conquered. (Mauri 2008, 60)

Catalan Sign Language (LSC), the sign language used by the deaf community in Catalonia (Spain), also shows a productive use of coordination. As we can see in the examples that follow in (5), in LSC, as in English, it is also possible to combine different syntactic categories.

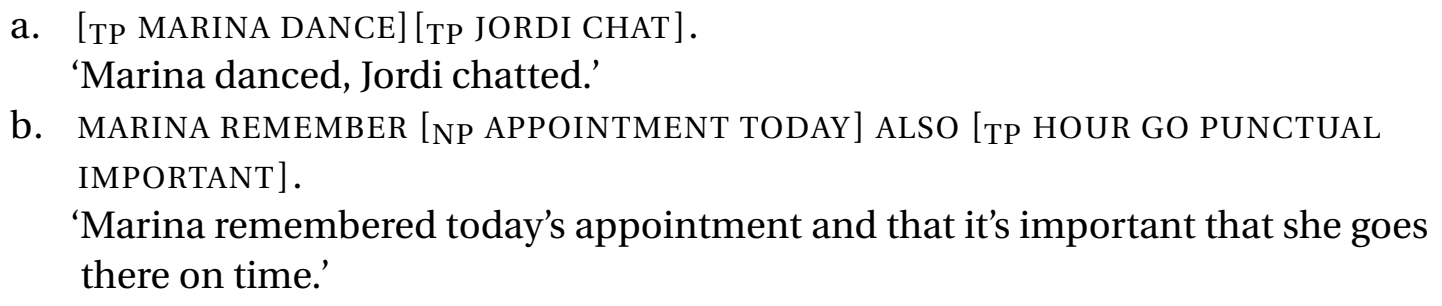

This paper aims to provide a syntactic analysis for conjunction in LSC, justifying a rightbranching asymmetric coordination structure that can also account for disjunctive and adversative coordination. In order to show that, in section 2 we will first explain how coordination in LSC is expressed. In section 3, we will introduce the tests that have been proposed to tell apart coordination and subordination in sign languages (SLs), applying those tests to LSC. Finally, in section 4, we will justify the need of having Coordination Phrase (CoP) as phrase for the whole coordination structure, and we will see the arguments that support an asymmetric and right-branching coordination structure. The LSC data presented in this paper have been collected from two native LSC signers through various elicitation techniques.

\section{Expression of coordination in LSC}

Coordination in LSC is primarily expressed asyndetically, through the use of non-manual markings (NMMs) such as head and body movement, and use of space. ${ }^{2}$ In particular, as we can see in Figure (1), head lean (hl) and body shift (bs) towards the ipsilateral side of the vertical plane in front of the signer spread over the verb BUY. This is the side that corresponds to the dominant hand of the signer, where the verb is also produced. In opposition to the ipsilateral side, there is the contralateral one corresponding to the one of the non-dominant hand of the signer. As we will see next, the contrast between these two sides is crucial to express coordination. It is expressed through the movement of the body and the head and

\footnotetext{
${ }^{1}$ They might be cases of CP coordination that involve the presence of Topics or Foci, but I will not enter this matter here.

${ }^{2} \mathrm{Head}$ and body movement and use of space will not be considered overt coordinators in LSC, differently than how Davidson (2011) treats the movement of the torso in coordination in American Sign Language (ASL). She glosses it as COORD-SHIFT.
} 
the use of the space. Similar NMMs and use of space are found not only in conjunctive but also in disjunctive and adversative coordination.

Figure 1: NMMs (verb BUY in LSC)

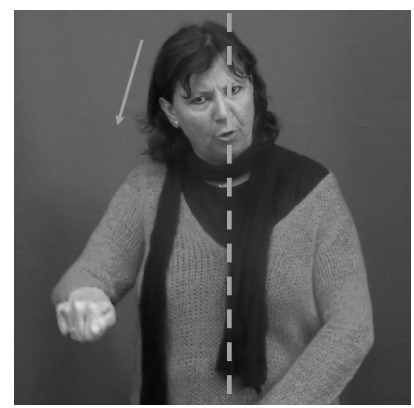

In LSC, it is possible to find instances of syndetic coordination, too. In conjunction there are two main overt markers used, glossed as ALSO, see Figure (2), and PLUS. PLUS can come in two different forms, as shown in Figure (3) and Figure (4).

Figure 2: ALSO

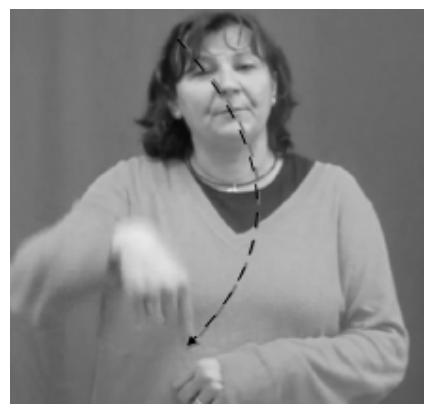

Figure 3: PLUS_a

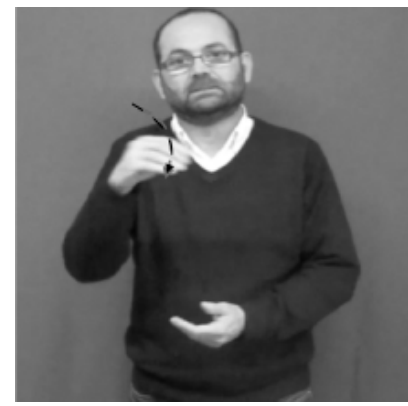

Figure 4: PLUS_b

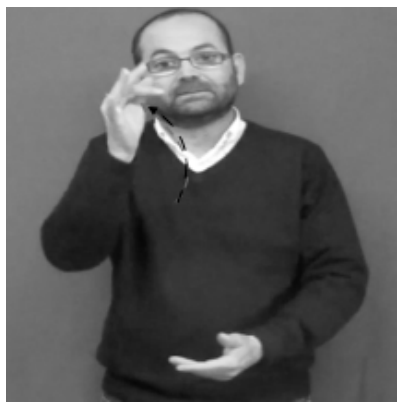

In example (6) below, we can see two examples of conjunction expressed in LSC through the combination of NMMs, use of space and the overt sign ALSO which appears between the conjuncts. In (6a), the conjuncts are identified through the contrast in the use of space, and the presence of the overt marker ALSO produced between them. The first two conjuncts are primarily composed of body-anchored signs, the second one entirely, but they are contrasting thanks to the production of the verb BAKE in the contralateral side of the space in the first conjunct. The third conjunct, as the second one, is produced with head lean (hl) towards the ipsilateral side, but the presence of a single head nod (shd) at the end of the second conjunct and the use of ALSO mark the boundary between the last two. When two or more conjuncts are coordinated, there is no default location for the first conjunct and no neat alternation of the NMMs between the conjuncts. (6b) also shows that the use of the contralateral and ipsilateral side alternates between the two conjuncts, and even though in the last one the head lean is kept towards the same side of the previous one, the contrast is marked through the use of the space. Moreover, we can see that ALSO and PLUS can be combined in the same sentence to coordinate more than two conjuncts and this aspect will be important to shape the structure of coordination in section 4 . 

hl ipsil. sp.contral
$\underline{\operatorname{shn}}$
hhn
hl ipsil.
$\underline{\text { shn }}$

MARC CAKE $\overline{\text { BAKE }} \overline{\text { ALSO MARINA PIZZA EAT }} \overline{\text { ALSO IX-2 }} \overline{\text { SANDWICH PREPARE. }}$

'Marc baked a cake, Marina ate a pizza, and you prepared a sandwich.'

$\begin{array}{lll}\text { bp.contral. } & \frac{\text { space ipsil. }}{\text { bl+bs ipsil. }} \text { re } & \text { sp.contral. } \\ \text { hl ipsil. }\end{array}$

b. $\overline{\text { HOME MARINA GO }} \overline{\text { ALSO TICKET CINEMA BUY PLUS }} \overline{\text { SON SCHOOL BRING. }}{ }^{3}$

'Marina went home, she bought the ticket for the cinema and she brought her son to school.'

After showing how coordination is expressed in LSC, let us present the tests that can confirm the presence of this structure in opposition to subordination.

\section{Tests to identify coordination}

Differently than spoken languages, in SLs the distinction between coordination and subordination is harder to detect due to the absence of complementizers such as "that", for example. It is important, then, to apply some tests. The main ones that have been proposed for SLs by Tang and Lau (2012),Van Gijn (2004) and Göksel and Kelepir (2016), among others, focus on the following aspects:

- scope in yes/no questions

- distributional dependency

- position of prosodic boundaries

As for the first test, Tang and Lau (2012) suggest, for Hong Kong Sign Language (HKSL), to look at the scope of the confirmation tag at the end of a yes/no question. If this final element can scope over the conjuncts that precede it, then the presence of coordination is confirmed. In (7) we can see an example from LSC.

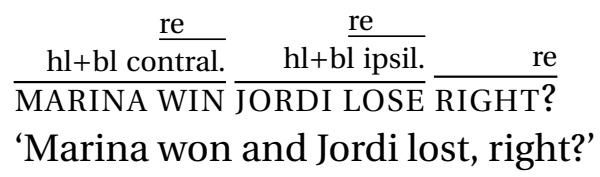

In (7), the fact that the confirmation tag RIGHT is scoping over both conjuncts confirms the presence of coordination in LSC. Moreover, the NMMs spreading over the two conjuncts are a further proof.

The other two tests listed above can only be applied to TP coordination and were initially proposed respectively by Van Gijn (2004), for Sign Language of the Netherlands (NGT), and by Göksel and Kelepir (2016), for Turkish Sign Language (TID), to establish the presence of subordination in opposition to coordination. For LSC, we are going to use them to exclude the presence of subordination in favor of coordination.

The first one consists of looking at distributional dependency. As we can see in (8) for LSC, the two TP conjuncts constitute two independent sentences that are not in a dependence relation with each other. Assuming that there is a salient referent for the pronoun used in the second conjunct, the two sentences can be correctly produced by themselves. This property supports the presence of coordination, together with the NMMs that, spreading over the conjuncts, help to identify the conjuncts and bundles them prosodically into a single sentence.

\footnotetext{
${ }^{3}$ The gloss re stands for "raised eyebrows".
} 
(8)

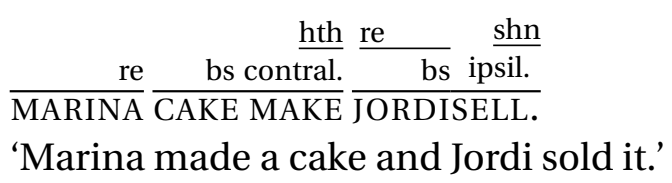

Looking at the NMMs found at the end of each conjunct constitutes the other test proposed by Göksel and Kelepir (2016), in line with a study made by Gökgöz and Arik (2011) on TİD. In order to exclude the presence of coordination, it is possible to examine the use of certain NMMs that have the function of prosodic boundaries such as head thrust (hth), eye-blink (eb) or single head nod (shn). As we can see in (8) and in (9), these NMMs are also found in LSC to mark the presence of coordination excluding instances of subordination, in which they are absent.

$$
\frac{\mathrm{re}}{\text { MARINA }} \frac{\underline{\mathrm{eb}}}{\text { DANCE }} \frac{\frac{\mathrm{re}}{\mathrm{hl+bl+} \text { sp.ipsil. }}}{\text { JORDI CHAT. }},
$$

Two more tests that have been proposed in the literature consist of checking the availability of Across-the-Board (ATB) movement and wide scope of negation. Both phenomena are not grammatical in LSC, and we attribute their ungrammaticality to the directionality of the coordination structure, as we will see in section 4.2. The tests successfully applied here are anyway enough to confirm that coordination is an attested structure in LSC.

In the next section, we will present the data that will motivate an asymmetric rightbranching structure for coordination in LSC.

\section{Towards a syntactic representation}

To represent coordination in LSC, we will adopt an asymmetric structure in which, following Munn (1987), conjuncts are specifiers and complements of a Coordination Phrase, more specifically CoP. In the next sections, we will go through the reasons for using a specific category for the whole coordination structure, and we will get into the details that justify the need of having a right-branching coordination structure, despite LSC being a head-final language.

\subsection{Why CoP}

In the literature, the coordinator is considered a head, but the debate is still open as to the category of the phrase to which this head belongs. For LSC, we will use CoP (Coordination Phrase). There are several reasons to choose a specific category for the syntactic representation of coordination. The first one relates to the types of categories that can be coordinated. Zhang (2010) points out that in English it is possible to coordinate different categories and not all of them need to saturate the verb. As we can see in (10), where the main verb is a phrasal verb, it is always the category of the first conjunct that needs to saturate the verb, but it is not the case for the category of the second one.

(10) a. You can depend on [NP my assistant] and [CP that he will be on time].

b. You can depend on [NP my assistant].

c. *You can depend on [CP that he will be on time]. 
From these data, Zhang (2010) proposes that the coordination structure takes as selected category the one of the first conjunct. For the examples in (10), it will be NP. In LSC, though, the category of each conjunct needs to saturate the verb, as we can see in (11).
a. MARINA REMEMBER [NP APPOINTMENT TODAY] ALSO [TP HOUR GO PUNCTUAL IMPORTANT].
'Marina remembered today's appointment and that it's important that she goes there on time.'
b. MARINA REMEMBER [NP APPOINTMENT TODAY]. 'Marina remembered today's appointment.'
c. MARINA REMEMBER [TP HOUR GO PUNCTUAL IMPORTANT].
'Marina remembered it's important that she goes there on time.'

For LSC, then, there is no way to tell whether it would be the first or the second conjunct to give the category to the whole structure. Therefore, the generalization by Zhang (2010) does not hold for LSC.

Another argument that supports the use of CoP is the fact that the presence of coordination in LSC, differently than spoken languages, can be detected from the beginning of the sentence. In English, for example, it is necessary to wait until the production of the coordinator "and" or until the production of the second conjunct in order to understand that we are dealing with coordination. In LSC, instead, the NMMs described in section 2 make clear from the beginning of the sentence that the structure that will be produced is a coordinated one. CoP, then, can be adopted as the category that marks the presence of coordination since the beginning of the sentence.

Moreover, thanks to the spec-head agreement in CoP, the coordination features in the first conjunct, realized via NMMs, are licensed. The ones in the second conjunct and the others have a variable realization, but they are always in prosodic contrast with the ones of the previous one, at least in the orientations of the body or of the space.

In order to provide a proper structure for coordination in LSC, though, it is also crucial to consider its directionality, namely whether it is right-branching or left-branching.

\subsection{Directionality of CoP}

An important question to ask regarding coordination structure is its directionality. Zhang (2010) argues that it depends on the position of the coordinator with respect to the conjuncts. When the coordinator appears between two conjuncts, it is possible to find two types of coordination:

- prepositive: the coordinator belongs to the second conjunct $([[\alpha][\& \beta]])$

- postpositive: the coordinator belongs to the first conjunct ([[ $\alpha \&][\beta]])$

In order to establish the position of the coordinator, Haspelmath (2007) proposes to look at intonational grouping and check whether a prosodic break is produced before or after the coordinator. In English, for example, only (12a) is grammatical, where the prosodic break is produced before "and", while (12b) is not. In the two examples, the comma marks the prosodic break.

(12) a. The son got married, and the daughter graduated.

b. *The son got married and, the daughter graduated. 
Based on the data in (12), in English, it is concluded that the coordinator belongs to the second conjunct, and therefore it shows prepositive coordination. We can get to the same conclusion also looking at the prosodic contour in LSC. As we can see in (13), a prosodic break is produced before the coordinator ALSO, which is realized in a prosodic continuum with the second conjunct.
$\frac{\text { hl contral. }}{\text { MARINA ICE-CREAM, }} \frac{\mathrm{hl} \text { ipsil. }}{\mathrm{hl} \text { ipsil }}$
'Marina paid for an ice-cream and for a chocolate.'

In LSC, as in English, the coordinator belongs to the second conjunct and therefore there is prepositive coordination, as well.

Depending on the position of the coordinator, Zhang (2010) argues that when there is prepositive coordination, in which the coordinator belongs to the second conjunct, that language needs a right-branching coordination structure, like in Figure (5); if it belongs to the first one, instead, realizing postpositive coordination, a left-branching coordination structure is necessary, like the one in Figure (6).

Figure 5: Right-branching coordination

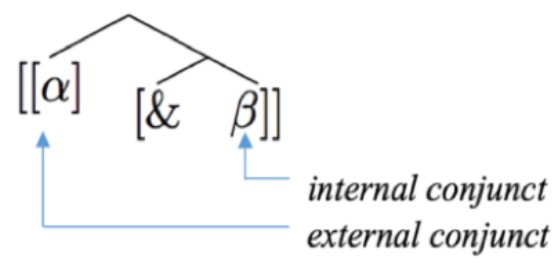

Figure 6: Left-branching coordination

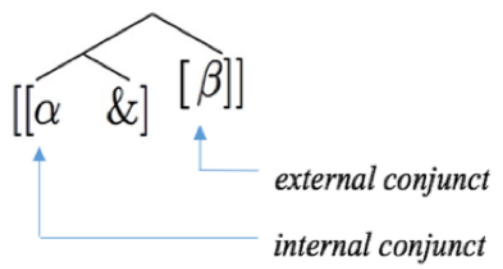

In LSC, we would expect the presence of a left-branching coordination structure, since it is a head-final language, but, based on Zhang's (2010) generalization, it is necessary to propose a right-branching analysis. Further data such as the impossibility of having Across-the-Board (ATB) extraction, the absence of wide scope of negation over both conjuncts and the properties of the directionality of gapping in coordination support this conclusion. In these three structures, the position of the conjuncts is crucial in determining the grammaticality of the sentence. As we can see in (14), ATB extraction in LSC is not allowed: it is not possible to ask a question about an element in both conjuncts that happens to be the same.

*MARINA $t_{i}$ BUY, JORDI $t_{i}$ READ WHAT $i$ ?

'What did Marina buy and Jordi read?'

Assuming the analysis in two steps by Wilder (1994) for the derivation of ATB structures, the wh-movement happens first within each conjunct separately, and then the wh-element in the second conjunct gets deleted via ellipsis, as we can see in (15).

\section{(15) [Which book does Peter like ] and [which book does Susan hate]? (De Vries 2017, 1)}

The use of a right-branching coordination structure, then, like the one in (16), can justify the ungrammaticality of the example in (14) in LSC. The position of the first conjunct, which contains the ellipsis site, is too high. Deletion cannot be licensed by the material in the second conjunct that appears in a lower position. The WHAT in the second conjunct is too low to license the one that gets deleted in the first conjunct. 


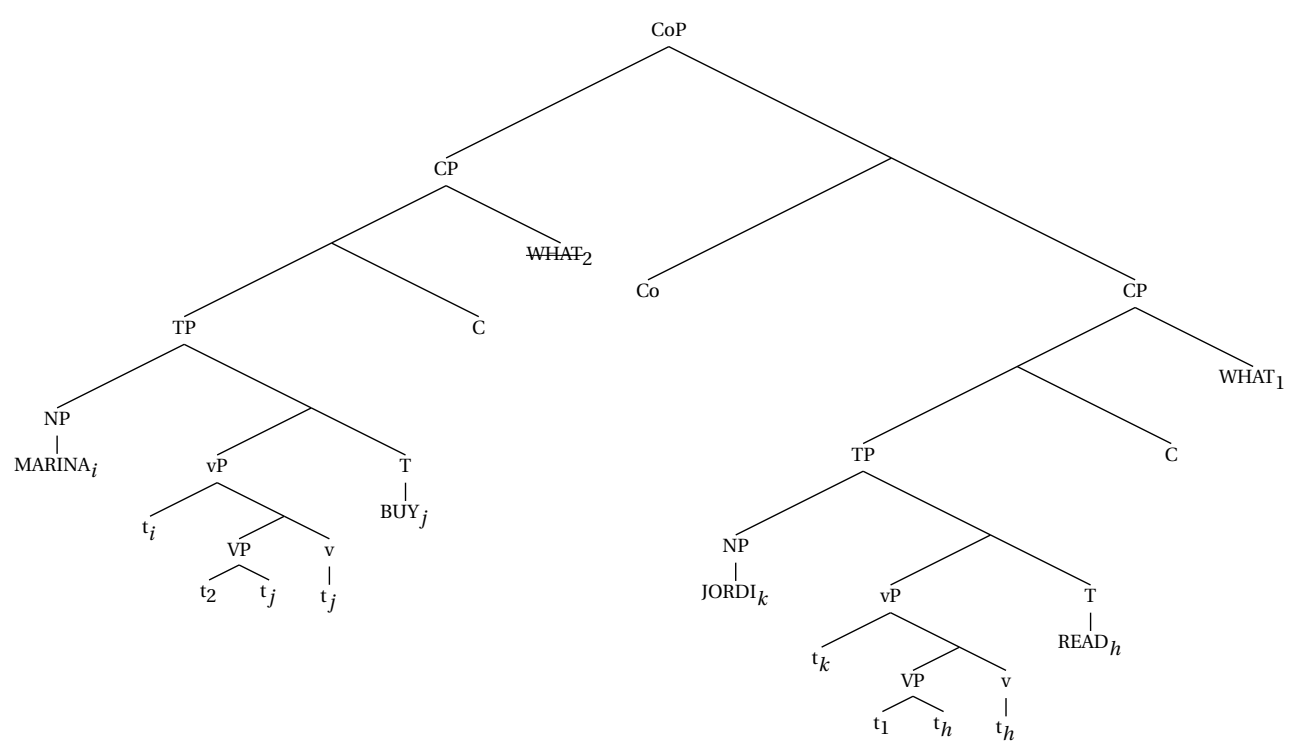

The only way of asking about an element in both conjuncts is through the production of an overt wh-element in the right periphery of both conjuncts, like in (17).

$$
\begin{aligned}
& \frac{\mathrm{fe}}{\mathrm{bl}+\mathrm{hl}+\text { space contral. }} \\
& \frac{\mathrm{fe}}{\text { MARINA BUY WHAT }} \frac{\mathrm{bl}+\mathrm{hl}+\text { space ipsil. }}{\text { JORDI READ WHAT? }}{ }^{4} \\
& \text { 'What did Marina buy and Jordi read?' }
\end{aligned}
$$

We can further prove the need of a right-branching coordination structure for LSC if we consider the scope of negation. In LSC, negation is placed in the right periphery of the sentence and structurally it is found in a negative phrase (NegP) above TP (vP > TP > NegP > CP; cf. Pfau and Quer (2007)). As we can see in (18), it is not possible to negate both conjuncts with the negation placed in the right periphery of the whole coordinated sentence.

\section{(18)}

*TEACHER FOOD COOK SANDWICH BUY NOT.

Intended 'The teacher didn't cook the food and didn't buy a sandwich.'

Similar to what we observed for the licensing of the deletion of WHAT in ATB extraction, in (18) the negation in the second conjunct is too low to negate the first conjunct, as we can see in the representation in (19). The ungrammaticality of (18) is, again, due to the presence of a right-branching structure.

\footnotetext{
${ }^{4}$ The gloss fe stands for "furrowed eyebrows".
} 


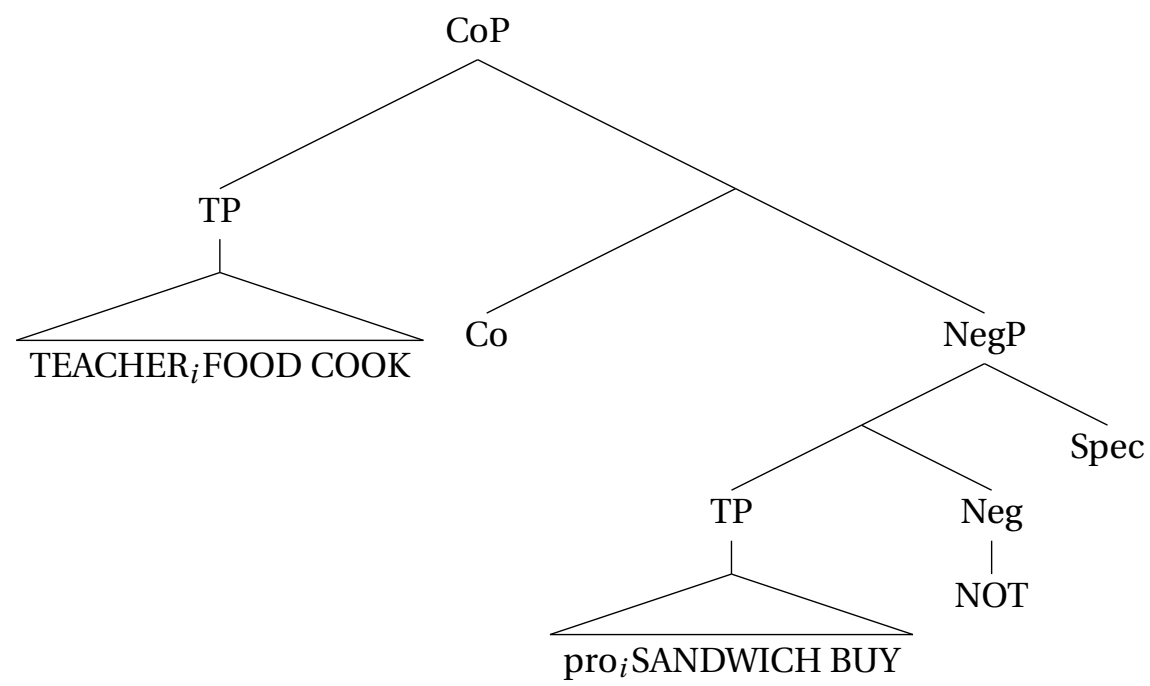

We can see in (20) that only the production of the negation in both conjuncts can repair the ungrammatical sentence in (18).

(20) TEACHER COOK FOOD NOT SANDWICH BUY * (ALSO-NOT) ${ }^{5}$

'The teacher didn't cook the food and didn't buy a sandwich.'

Finally, the directionality of gapping leads to the same conclusion. LSC, despite being an SOV language, gaps forward, realizing the SOV-SO order (Zorzi 2018). Assuming that the gapped site needs to be licensed by the material in the antecedent conjunct, the second conjunct needs to be lower than the first one, as in the representation of example (21) in (22).

$\frac{\mathrm{re}}{\text { MARINA }} \frac{\mathrm{hl} \text { contral. }}{\text { COFFEE PAY }} \frac{\text { re }}{\text { JORDI CROISSANT PAY. }}$

'Marina paid for a coffee and Jordi for a croissant.'

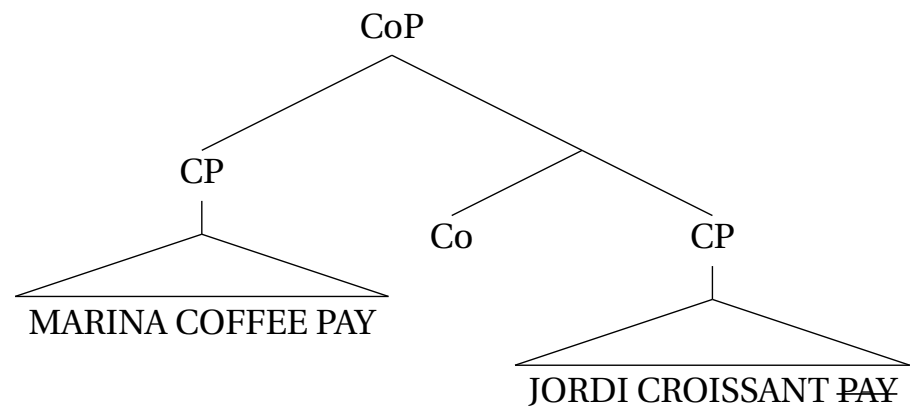

From the properties reviewed that confirm that LSC needs to adopt a right-branching coordination structure whose category is CoP, let us examine the representation of (6a), repeated here in (23). In the representation in (24), CoP is the category of the whole structure, the conjuncts are specifiers and complements of $\mathrm{CoP}$ and, due to the possibility of having two different coordinators as we saw in example (6b), the two CoPs are differentiated.

\footnotetext{
${ }^{5}$ In this example the NMMs are omitted because they are irrelevant to the discussion.
} 
(23) $\frac{\text { hl.ipsil. sp.contral. }}{\text { MARC CAKE }} \frac{\text { hl. }}{\text { BAKs }} \frac{\text { shil. }}{\text { ALSO MARINA PIZZA EAT }} \frac{\text { hl.ipsil. }}{\text { ALSO IX-2 }} \frac{\text { sh }}{\text { SANDWICH PREPARE. }}$

'Marc baked a cake, and Marina ate a pizza, and you prepared a sandwich.'

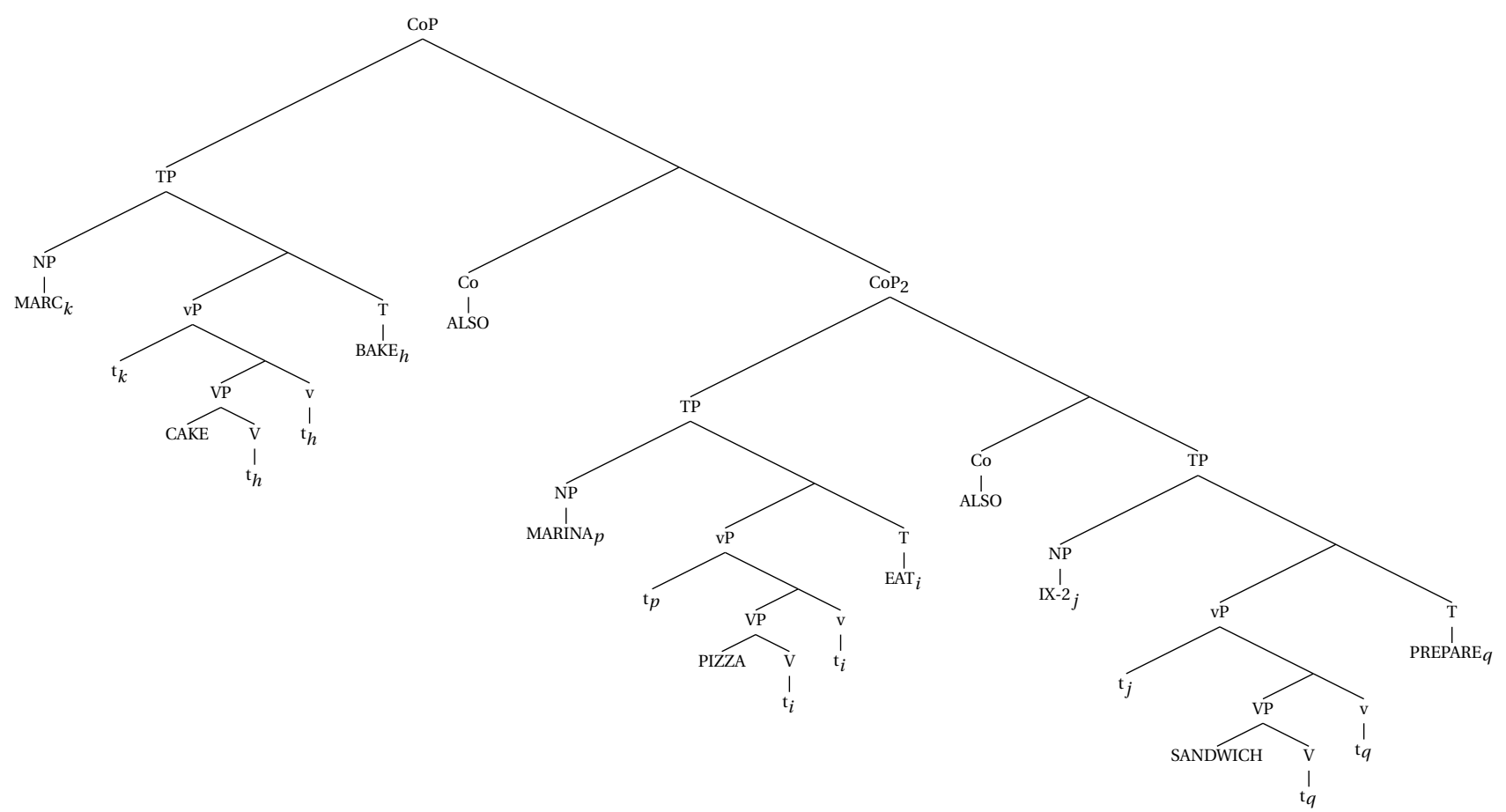

\section{Conclusions}

In this paper, we presented how coordination is expressed in LSC, mainly focusing on conjunction of TPs. Moreover, we provided a syntactic representation in which CoP is the syntactic category for the whole structure. Following Munn (1987), the specifier and complement of CoP host the conjuncts. This syntactic structure is meant to be used also for other types of coordination in LSC, such as disjunctive and adversative coordination.

\section{Acknowledgments}

I would like to thank Delfina Aliaga Emeterio and Santiago Frigola Segimon for their fantastic job as LSC consultants. I would also like to thank the audience of FEAST 2018 and CreteLing Workshop "Sign language grammars through the formal and experimental glass" where these data were presented. This research has been funded by the Spanish Ministry of Economy and Competitiveness "ClauseCombi2" FFI2012-36238, BES2013062848, "GramRefLSC FFI2015-68594-P, Govern de la Generalitat de Catalunya (2014 SGR 698) and SIGN-HUB project, which has received funding from the European Union's Horizon 2020 research and innovation programme under grant agreement No 693349. 


\section{References}

Davidson, Kathryn. 2011. "The nature of the semantic scale: Evidence from sign language research.” PhD thesis, University of California.

De Vries, Mark. 2017. “Across-the-Board phenomena.” In The Wiley Blackwell companion to syntax. Second edition. Ed. by M. Everaert and H. van Riemsdijk, 1-31. Wiley Blackwell.

Gökgöz, Kadir, and Engin Arik. 2011. "Distributional and syntactic characteristics of nonmanual markers in Turkish Sign Language (Turk İşaret Dili, TID)”. In MIT Working Papers in Linguistics 62. Proceedings of the 7th Workshop on Altaic Formal Linguistics, 63-78.

Göksel, Asli, and Meltem Kelepir. 2016. "Coordination and subordination.” In A matter of complexity: Subordination in sign languages, ed. by Annika Herrmann, Roland Pfau, and Markus Steinbach, 65-94. Berlin: Mouton De Gruyter.

Haspelmath, Martin. 2007. "Coordination." In Language typology and syntactic description, vol. II: Complex constructions, ed. by Timothy Shopen, 1-51. Cambridge: Cambridge University Press.

Mauri, Caterina. 2008. Coordination relations in the Languages of Europe and Beyond. Berlin: Mouton de Gruyter.

Munn, Alan. 1987. "Coordinate structure and X-bar theory". McGill Working Papers in Linguistics 4 (1): 121-140.

— . 1993. "Topics in the syntax and semantics of coordinate structures". PhD thesis, University of Maryland.

Pfau, Roland, and Josep Quer. 2007. "On the syntax of negation and modals in German Sign Language (DGS) and Catalan Sign Language (LSC).” In Visible variation. Comparative studies on sign language structure. Ed. by Pamela Perniss, Roland Pfau, and Markus Steinbach, 129-161. Berlin: Mouton de Gruyter.

Sag, Ivan, Gerald Gazdar, Thomas Wasow, and Steven Weisler. 1985. "Coordination and how to distinguish categories”. Natural Language and Linguistic Theory 4 (3): 117-171.

Tang, Gladys, and Prudence Lau. 2012. “Coordination and subordination.” In Sign language: An international handbook, ed. by Roland Pfau, Markus Steinbach, and Bencie Woll, 340365. Berlin: Mouton De Gruyter.

Van Gijn, Ingeborg Caren. 2004. The quest for syntactic dependency. Sentential complementation in Sign Language of the Netherlands. Utrecht: LOT.

Wilder, Chris. 1994. "Coordination, ATB and ellipsis". GAGL 37:291-329.

Zhang, Niina Ning. 2010. Coordination in syntax. Cambridge: Cambridge University Press.

Zorzi, Giorgia. 2018. “Gapping vs. VP-ellipsis in Catalan Sign Language.” FEAST 1:70-81. 